\title{
Vision and Cerebellum: Evaluating the Influence of Motor Control on Cognitive Execution through Multiscale Wavelet Entropy
}

\author{
Giacomo Veneri ${ }^{1,2, *}$, Francesca Rosini ${ }^{1}$, Alessandra Rufa ${ }^{1,2}$ \\ ${ }^{1}$ Department of Neurological and Behavioral Science - University of Siena, Siena, 53100, Italy \\ ${ }^{2}$ Eye tracking \& Visual Applications Lab - University of Siena, Siena, 53100, Italy
}

\begin{abstract}
Active vision integrates the information coming, through the retina, from the outside world with that generated internally by the brain, especially by eye movements (saccades). We investigate the influence that the eyes motor control might play on visual search. Thirteen cerebellar patients and twenty-five healthy subjects performed a high cognitively demanding task (Trail Making Test), we correlated the performance of eye motor control with the abilities to perform the task efficiently, and we found a strict correlation between the two factors on patients. The experimental evidence supports an indirect role of the cerebellum on cognitive performance due to noise feedback.
\end{abstract}

Keywords Selective Attention, Motor Control, Cerebellum, Cognition, Optimal Control Theory

\section{Introduction}

Since the visual system has limited computational resources $[43,8]$, it is necessary to limit detailed processing to selected aspects of the input: for this reason, humans select some regions from the scene to process in depth limited information, then move the eyes to a location. This mechanism is usually referredto as "selective attention"[32]. Selective attention is managed by two competitive mechanism: saliency of the scene (bottom-up) and internal intention (top-down) (see Figure 1). These two mechanisms contribute to build the priority (maybe Bayesian;[27, 19, 44]) map[12] which suggests the next location to explore; after that, the eye movement (saccade) is planned and controlled through the cerebellum-cerebral loop[29]. Selective attention and eye movements cooperate to the perception of a stable visual world (see[33] for premotor theory of attention; see[22] for corollary discharges), we refer to this vision as "active vision".

Since the eye movement is influenced by noise[4], saccades are generated with movement profiles that minimize the impact of all forms of motor noise[30]; then, the question is "could sensory-motor noise feedback influence the selective attention?" (Figure 1). To answer this question we studied the visual exploration made by patients affected by cerebellar atrophy: it is well known that patients affected by

\footnotetext{
* Correspondingauthor:

g.veneri@unisi.it (Giacomo Veneri)

Published online at http://journal.sapub.org/ijbcs

Copyright $\left({ }^{\circ} 2012\right.$ Scientific \& Academic Publishing. All Rights Reserved
}

cerebellar disease have reduced capacities to control eye movement $[47,21]$; indeed, the neuronal circuitry of the cerebellum (CBLM) is thought to encode internal models that reproduce the dynamic properties of body parts[14]. These models control the movement allowing the brain to precisely control the movement without the need for sensory feedback $[3,16,18]$. The CBLM is linked to the cerebral cortex via two-stage feedforward and feedback systems[20]. Input from cerebral cortical regions terminates on nuclei in the basis pontis[29], which in turn convey mossy fibreefferents to the CBLM. Feedback projections from the cerebellar cortex travel via the deep cerebellar nuclei and terminate in the thalamus, which then sends projections back to the cerebral cortex $[17,45]$. This loop is a forward model avoiding direct input from the sensory apparatus[11] like an open adaptive filter[15]. Then, there are sufficient findings to account for a dominant role of the CBLM on motor control, while, the hypothesis that CBLM influences cognitive functionsremains unclear (see[37, 36] for a review).Various authors have argued a role of CBLM in visual-spatial functions:[46] and[42] supported the hypothesis that the CBLM is involved on spatial rotation.[2] suggested that only mid cerebellar regions (cerebellar vermal structures such as the pyramid) are indirectly involved in covert visual attention via oculomotor control mechanisms. On the contrary, experiments made by[5] failed to support the hypothesis that the CBLMis involved with attentional set shifting.

In our research, we aimed to study the role of cerebellum in visual search: we correlated the motor entropy to visual search performance, and we found that patients' motor control might play a role to select the next region to explore. 


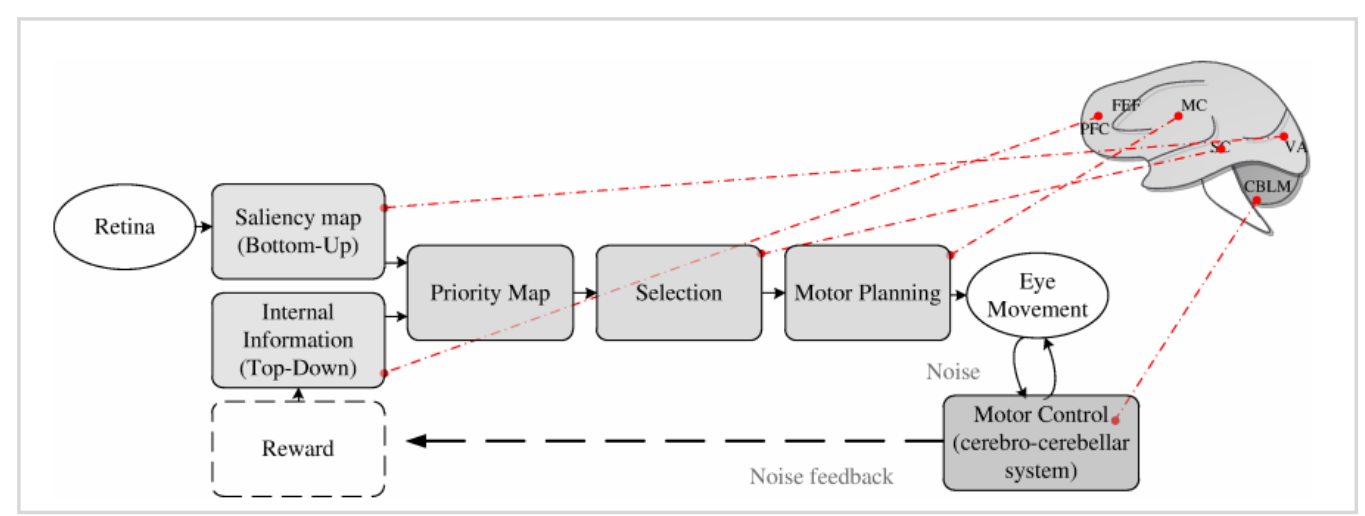

Figure 1. Attention and motor control. Selective attention is controlled by two cognitive top-down factors, such as knowledge, expectation and current goals, and bottom-up factors that reflect sensory stimulation. These two factors contribute to construct a priority map which guides next eye movement. After that, attention and motor planning collaborate to perform a stable vision of the world. Motor execution is controlled by cerebro-cerebellar loop; the effort on managing this movement and the noise to make a stable vision may influence next area selection (dashed line) such as an optimal control system. From the physiological point of view, Saliency Map is built on Visual Area (VA; see[1, 25]), Top-Down information is encoded on Prefrontal Cortex and Frontal Eye Fields (PFC and FEF; see[23]), these two maps are integrated on Superior Colliculus (SC; see[24, 9]), the motor command is executed and controlled through the cerebellum-cerebral loop (CBLM; see[20]). The optimal control vision, here described, could modulate the reward system[13] to minimize the effort on controlling movements. \{TC " 1 Attention and motor control. Selective attention is controlled by two cognitive top-down factors, such as knowledge, expectation and current goals, and bottom-up factors that reflect sensory stimulation. These two factors contribute to construct a priority map which guides next eye movement. After that, attention and motor planning collaborate to perform a stable vision of the world. Motor execution is controlled by cerebro-cerebellar loop; the effort on managing this movement and the noise to make a stable vision may influence next area selection (dashed line) such as an optimal control system. From the physiological point of view, Saliency Map is built on Visual Area (VA; see[1, 25]), Top-Down information is encoded on Prefrontal Cortex and Frontal Eye Fields (PFC and FEF; see[23]), these two maps are integrated on Superior Colliculus (SC; see[24, 9]), the motor command is executed and controlled through the cerebellum-cerebral loop (CBLM; see[20]). The optimal control vision, here described, could modulate the reward system[13] to minimize the effort on controlling movements." $\backslash$ f f $\}$

\section{Method}

We used a highly cognitive demanding task, namely the trail making test[6, TMT], in which subjects were asked to follow an alphanumeric sequence with their gaze. The trail making stimulus was a pop-up high contrast image consist ing of a sequence of numbers and letters (1-A-2-B-3-C-4 $-D-5-E$ ) arranged in an unpredictable manner[40]. The $T M T$ is particularly suitable for studying selective attention, as it does not require any explicit feedback by subjects, and the test performance can be evaluated by a computational method.

\subsection{Subjects}

Eight SCA2 patients, a mixed group of five patients with undefined genetic cerebellar ataxia (NDC) and 25 healthy subjects were enrolled in the study. All were in the age range $25-45$ years. Subjects were seated at viewing distance of 78 $\mathrm{cm}$ from a 32" colour monitor $(51 \mathrm{~cm} \times 31 \mathrm{~cm})$. Eye position was recorded using an ASL 6000 system, which consists of a remote-mounted camera sampling pupil location at $240 \mathrm{~Hz}$. A 9-point calibration and 3-point validation procedure was repeated several times to ensure all recordings had a mean spatial error of less than $0.3 \mathrm{deg}$. Head movements were restricted using chin rest and bite.

\subsection{Gaze Evaluation}

Numbers and letters were sampled as a pre-defined rectangular region of interest (ROI) centred on letters and numbers and having widths and heights merging from $80 \times 80$ pixels to $100 \times 100$ pixels. The same ROI spatial distribution was pre-defined for all tasks. Fixations were calculated using a dispersion-based algorithm proposed by Salvucci[34] and adapted by Veneri-Piu[39] and saccades by Fisher[13] algorithm. The minimum duration of fixation was set at $50 \mathrm{~ms}$, the maximum dispersion to identify fixations was at one degree and the saccade threshold at $30 \mathrm{deg} / \mathrm{sec}[21]$.

We evaluated two types of indicators: motor control noise and test performance. To give a quantitative value of motor noise during the visual search, we used the wavelet entropy, which provided a value of gaze variability[4]. Entropy was calculated through coif 2 wavelet[38] over $x$ and $y$ axis $\left(E_{x}, E_{y}\right)$ and averaging the two values $\left(E=\left(E_{x}+E_{y}\right) / 2\right)$.

Cognitive performance was evaluated calculating the Euclidean distance for each fixation to next target $(D T)$ or to nearest ROI $(D N)[41]$.

\subsection{Statistics}

ANOVA was used to find the difference among the groups; post-hoc analysis was performed by the Sidak procedure for multiple Student's $t$ tests. Kolmogorov-Smirnov test was used to evaluate the assumption of normal distribution. Correlation was evaluated through parametric Pearson test and non-parametric Spearman test.Descriptive statistics are given as the mean \pm standard deviation or median $(25-75 \%$ interquartile range) as appropriate. $\mathrm{p}<0.05$ was considered significant.

\section{Results}

The goal of the wavelet entropy indicator was to estimate the motor control noise during the task: Figure 2.a shows the 
scatter diagram of $E_{x}, E_{y}$ of the groups. A qualitative analysis of the graph shows an increase of entropy from $C T R L$ to $N D C$ to $S C A 2$. ANOVA reported significant difference $(\mathrm{F}(2,35)=18.32, \mathrm{p}<0.001)$ on $E=\left(E_{x}+E_{y}\right) / 2$ and post-hoc Sidak procedure confirmed significant difference between CTRL-SCA2 ( 2 ( CTRL-SCA2 $\left._{2}<0.001\right)$ and NDC-SCA2 $\left(\mathrm{p}_{\mathrm{NDC}-\mathrm{SCA} 2}=0.018\right)$; no significant difference was found between $C T R L-N D C\left(\mathrm{p}_{\mathrm{CTRL}-\mathrm{NDC}}=0.28\right)$. Indeed, $N D C$ is a small mixed group where the disease is not explicit and provided a useful reference to study the mechanism (Figure 2.b).

To study the scan path made by subjects, we evaluated the ongoing distance to target for each fixation $(D T)$; $D T$ provided an indicator to understand the ability of humans to converge to the target. ANOVA reported significant difference among groups $(F(2,35)=9.476, \mathrm{p}<0.01)$ and post-hoc Sidak procedure confirmed significant difference betweenCTRL-SCA2 ( $\left.\mathrm{p}_{\mathrm{CTRL}-\mathrm{SCA} 2}<0.01\right), C T R L-N D C$ ( $\mathrm{p}_{\mathrm{NDC}-\mathrm{SCA} 2}$ $\leq 0.01)$; no significant difference was found between $S C A 2-N D C\left(\mathrm{p}_{\mathrm{SCA} 2-\mathrm{NDC}}=0.622\right)$.

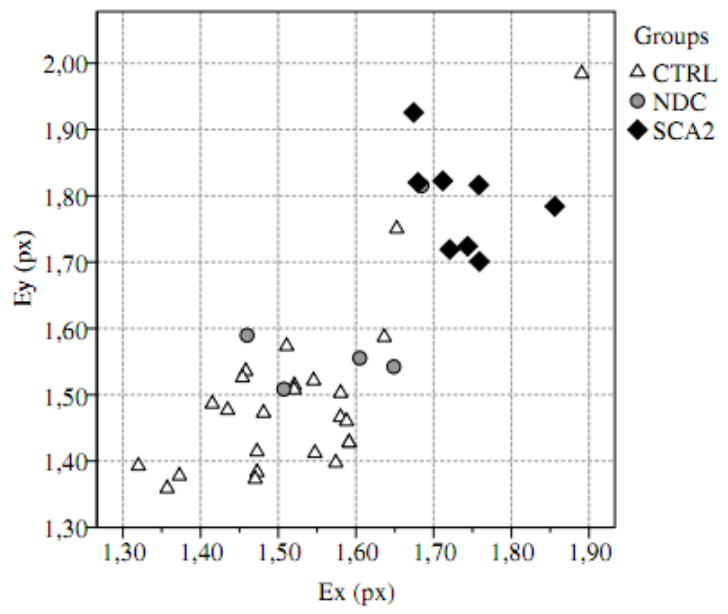

(a)

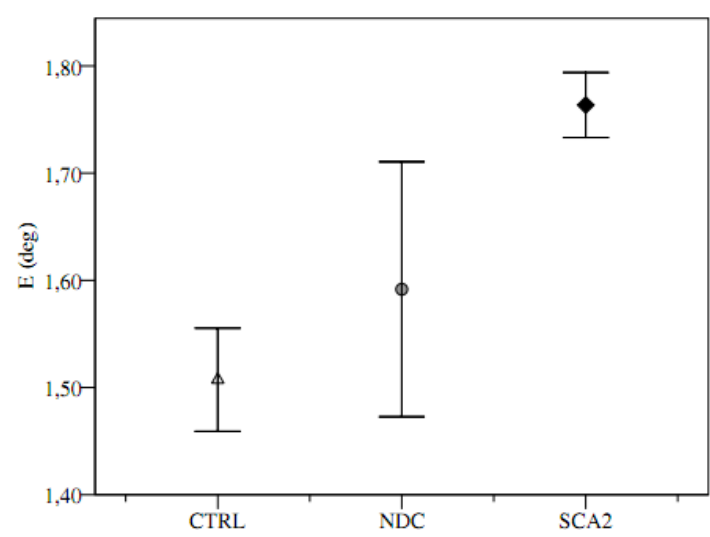

(b)

Figure 2. (a) Wavelet entropy over $x$ and $y$ for patients and healthy subjects. (b) Significant difference was found on wavelet entropy between healthy subjects $(C T R L)$ and $S C A 2$ patients; $N D C$ patients are a mixed group not easy to identify, but confirmed the high entropy eye motor control. \{TC "2 (a) Wavelet entropy over $\$ x \$$ and $\$ y \$$ for patients and healthy subjects. (b) Significant difference was found on wavelet entropy between healthy subjects $(C T R L)$ and SCA2 patients; $N D C$ patients are a mixed group not easy to identify, but confirmed the high entropy eye motor control." \f $\mathrm{f}$ \}
Pearson and Spearman test reported correlation between $E$ and $D T$ for $N D C$ patients ( $\mathrm{p}<0.05, \rho=0.892$, Figure $4 . \mathrm{a})$, and correlation for $S C A 2$ patients $(p<0.05, \rho=0.736$, Figure 4.b) not confirmed by Spearman $(\mathrm{p}=0.18)$. No correlation was found for $C T R L$ subjects $(\mathrm{p}=0.43)$.

Our preliminary conclusion was that motor control noise might influence test performance. To study why and how this effect could be valuable, we analysed distance to the nearest $R O I(D N)$; $D N$ measured the effect of target averaging, which is a strategy to select a region among available targets[35]. $D N$ reported the same characteristic of $D T$ $\left(\mathrm{p}_{\mathrm{CTRL}-\mathrm{SCA} 2}<0.001, \mathrm{p}_{\mathrm{CTRL}-\mathrm{SCA} 2}<0.01, \mathrm{p}_{\mathrm{NDC}-\mathrm{SCA} 2}=0.89\right)$, but not correlation with $E$ (Figure 3 ).

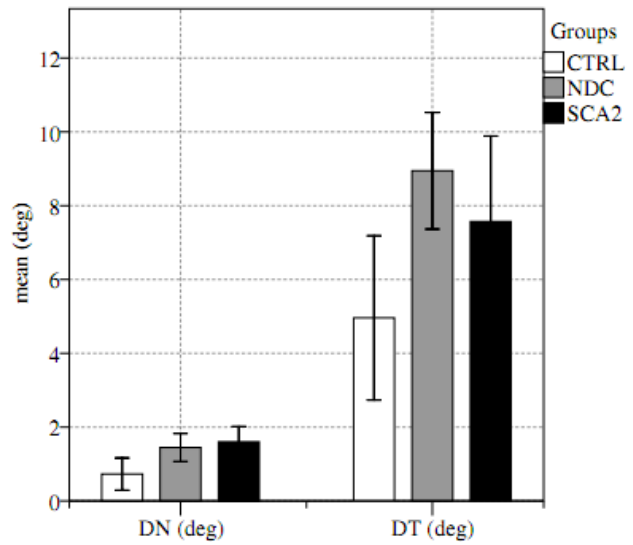

Figure 3. Exploration strategies. Significant differences of exploration strategies were found between patients and subjects; patients performed the test with sparser fixation (target averaging)

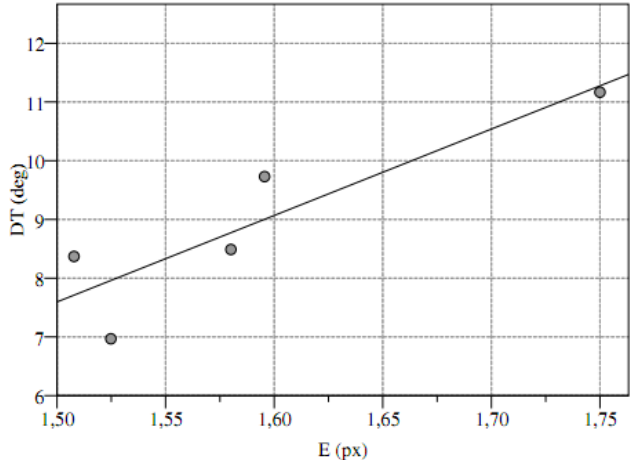

(a) DNC

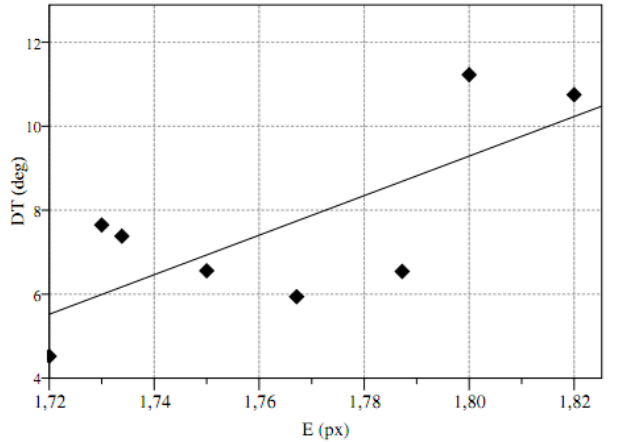

(b)SCA2 \{ TC " 4 Correlations between ongoing distance to target and entropy for patients SCA2 (a) and NDC (b)." \f f $\}$

Figure 4. Correlations between ongoing distance to target and entropy for patients SCA2 (a) and NDC (b) 
Table 1. Mean value and standard deviation (in brackets) of tests. $\{\mathrm{TC}$ "1 Mean value and standard deviation (in brackets) of tests." $\backslash \mathrm{f} t\}$

\begin{tabular}{|c|c|c|c|c|}
\hline Indicators & & CTRL & $N D C$ & $S C A 2$ \\
\hline $\begin{array}{c}\text { Group } \\
\text { dimension }\end{array}$ & $N$ & 25 & 5 & 8 \\
\hline Age & & $27-50$ & $40-55$ & $42-55$ \\
\hline Distance to nearest ROI (px) & $D N$ & $0.728( \pm 0.437)$ & $1.447( \pm 0.38)$ & $1.601( \pm 0.414)$ \\
\hline Distance to target (px) & $D T$ & $4.96( \pm 2.226)$ & $8.945( \pm 1.581)$ & $7.570( \pm 2.316)$ \\
\hline Entropy (px) & $E$ & $1.507( \pm 0.116)$ & $1.59( \pm 0.096)$ & $1.76( \pm 0.036)$ \\
\hline
\end{tabular}

\section{Discussion and Conclusions}

The significant difference of $D N$ and $D T$ (Table 1) and the correlation with $E$ provided evidence that patients performed the test with different strategies.

The role of the CBLMon in visual-spatial functions was argued by various authors[2, 37, 36, 46].[16] argued for a fascinating theory in which the CBLM may control mental activities by internal models[31]; indeed, the nature of humans is a continuous adaptation of action depending from previous action and stimulus. This hypothesis is systematically supported by recent application of optimal control theory;[27],[4] and[30] argued that humans' vision is an optimal mechanism minimizing the effect of motor or cognitive noise. Our findings are compatible with this hypothesis: patients preferred sparser fixations avoiding saccade directed to the target. This effect has been referred to as center-of-gravity (CoG). CoG occurs when targets are surrounded by non-targets, and the saccades, instead of landing at the designated target, land in the midst of the whole configuration. CoGwas considered a necessary mechanism to execute more efficient explorations[10].

The non-correlation of $D N$ with $E$ suggested that this mechanism was a strategy to minimize the effort to control saccade rather than a direct influence on saccade control.

Indeed, Cerebellum could influence selective attention (Top-Down) sending afferent information of noise (Figure 1) in order to minimize the functional cost of energy; this mechanism requires a reward for area that maximizes the efficiency. There are several finding[9] and experiments[26] addressing the superior colliculus (SC) in competitive stimulus selection[7];[28] and later[20], identified a strict collaboration between CBLM and SC, this collaboration could be the primary candidate to implement the mechanism hypothesized. Our hypothesis is that the reward idea, hypothesized by several authors[14], in which emotions and other factors could influence top-down attention, have to be integrated with afferent information coming from the cerebellum such as the active vision mechanism. During the last decade, attention has been studied independently of the motor control, but it is plausible that these two mechanisms influence each other in order to maximize efficiency.

\section{REFERENCES}

[1] E. Awh, K. M. Armstrong, and T. Moore. Visual and ocu- lomotor selection: links, causes and implications for spatial attention. Trends CognSci, 10(3):124-130, Mar 2006.

[2] B. Baier, M. Dieterich, P. Stoeter, F. Birklein, and N. G. $\mathrm{M} \Gamma \mathrm{jller}$. Anatomical correlate of impaired covert visual attentional processes in patients with cerebellar lesions. J Neurosci, 30(10):3770-3776, Mar 2010.

[3] J. S. Barlow. The cerebellum and adaptive control. Cambridge University Press, 2002.

[4] R. J. Beers. The sources of variability in saccadic eye movements. Journal of Neuroscience, 27(33):8757-8770, Aug 2007.

[5] A. Bischoff-Grethe, R. B. Ivry, and S. T. Grafton. Ce-rebellar involvement in response reassignment rather than attention. $\mathrm{J}$ Neurosci, 22(2):546-553, Jan 2002.

[6] C. R. Bowie and P. D. Harvey. Administration and interpretation of the trail making test. Nat Protoc, 1(5):2277-2281, 2006.

[7] C. D. Carello and R. J. Krauzlis. Manipulating intent: evidence for a causal role of the superior colliculus in target selection. Neuron, 43(4):575-583, Aug 2004.

[8] M. Carrasco. Visual attention: The past 25 years. Vision Research, pages 1-1, Apr 2011.

[9] J. Cavanaugh and R. H. Wurtz. Subcortical modula-tion of attention counters change blindness. J Neurosci, 24(50):11236-11243, Dec 2004.

[10] Cohen, E. H., Schnitzer, B. S., Gersch, T. M., Singh, M., \& Kowler, E. (2007). The relationship between spatial pooling and attention in saccadic and perceptual tasks. Vision Research, 47, 1907-1923.

[11] L. Fautrelle, C. Pichat, F. Ricolfi, C. Peyrin, and F. Bonnetblanc. Catching falling objects: the role of the cerebellum in processing sensory-motor errors that may influence updating of feedforward commands. an fmri study. Neuroscience, Sep 8;190:135-44. 2011 Jun 28 .

[12] J. H. Fecteau and D. P. Munoz. Salience, relevance, and firing: a priority map for target selection. Trends CognSci, 10(8):382-390, Aug 2006.

[13] B. Fischer, M. Biscaldi, and O. P. Saccadic eye movements of dyslexic adults. Neuropsychologia, 31:887-906, 1993.

[14] A. M. Graybiel. The basal ganglia: learning new tricks and loving it. CurrOpinNeurobiol, 15(6):638-644, Dec 2005.

[15] M. Ito. Bases and implications of learning in the cerebellum-adaptive control and internal model me-chanism. Progress in Brain Research, 148:95-109, 2005.

[16] M. Ito. Control of mental activities by internal models in the 
cerebellum. Nature Reviews Neuroscience, 9(4):304-313, Apr 2008.

[17] R. M. Kelly and P. L. Strick. Cerebellar loops with motor cortex and prefrontal cortex of a nonhuman pri-mate. Journal of Neuroscience, 23(23):8432-8444, Sep 2003.

[18] S. King, A. L. Chen, A. Joshi, A. Serra, and R. J. Leigh. Effects of cerebellar disease on sequences of rapid eye movements. Vision Research, 51(9):1064-1074, May 2011.

[19] К. Р. КГPrding and D. M. Wolpert. Bayesian integra-tion in sensorimotor learning. Nature, 427(6971):244-247, Jan 2004.

[20] P. Lefevre, C. Quaia, and L. M. Optican. Distributed model of control of saccades by superior colliculus and cerebellum. Neural Networks, 11(7-8):1175-1190, 1998.

[21] Z. D. Leigh R.J. The neurology of eye movements (Book/DVD). Fourth edition. New York: Oxford University Press, 2006.

[22] E. P. Merriam and C. L. Colby. Active vision in pa-rietal and extrastriate cortex. Neuroscientist, 11(5):484-493, Oct 2005.

[23] T. Moore and K. M. Armstrong. Selective gating of visual signals by microstimulation of frontal cortex. Na-ture, 421(6921):370-373, Jan 2003.

[24] D. Munoz and R. Wurtz. Fixation cells in monkey superior colliculus. ii. reversible activation and deactiva-tion. Journal of Neurophysiology, 70:576-589, 1993.

[25] D. K. Murphey and J. H. R. Maunsell. Behavioral detection of electrical microstimulation in different cor-tical visual areas. CurrBiol, 17(10):862-867, May 2007.

[26] S. P. Mysore and E. I. Knudsen. The role of a mid-brain network in competitive stimulus selection. Current Opinion in Neurobiology, In Press, Corrected Proof:-, 2011.

[27] J. Najemnik and W. S. Geisler. Optimal eye move-ment strategies in visual search. Nature, 434(7031):387-391, Mar 2005.

[28] L. M. Optican. A field theory of saccade generation: Temporal-to-spatial transform in the superior colliculus. Vision Research, 35(23-24):3313-3320, 1995.

[29] L. M. Optican and D. A. Robinson. Cerebel-lar-dependent adaptive control of primate saccadic sys-tem. Journal of Neurophysiology, 44(6):1058-1076, Dec 1980.

[30] L. C. Osborne. Computation and physiology of sen-sory-motor processing in eye movements. Current Opi-nion in Neurobiology, 21(4):623-628, Aug 2011.

[31] E. Perlov, L. T. van Elst, M. Buechert, S. Maier, S. Matthies, D. Ebert, B. Hesslinger, and A. Philipsen. H1-mr-spectroscopy of cerebellum in adult attention deficit/hyperactivity disorder. Journal of Psychiatric Re-search, 44(14):938 - 943, 2010.

[32] M. Posner. Orienting of attention. Quarterly Journal of Experimental Psychology, 32:3-25, 1980.

[33] G. Rizzolatti, L. Riggio, I. Dascola, and C. Umilth. Reo- rienting attention across the horizontal and vertical meridians: evidence in favor of a premotor theory of attention. Neuropsychologia, 25(1A):31-40, 1987.

[34] D. D. Salvucci and J. H. Goldberg. Identifying fixa-tions and saccades in eye-tracking protocols. In ETRA '00: Proceedings of the 2000 symposium on Eye tracking re-search \& applications, pages 71-78, New York, NY, USA, 2000. ACM.

[35] S. Schneider and W. Erlhagen. A neural field model for saccade planning in the superior colliculus: speed-accuracy tradeoff in the double-target paradigm. Neurocomputing, 44-46:623 - 628, 2002.

[36] Sergio and D. Sala. Cognition and the cerebellum. Cortex, 47(1):1 -, 2011.

[37] C. J. Stoodley and J. D. Schmahmann. Evidence for topographic organization in the cerebellum of motor control versus cognitive and affective processing. Cortex, 46(7):831-844, 2010.

[38] G. Veneri, P. Federighi, F. Rosini, A. Federico, and A. Rufa. Spike removal through multiscale wavelet and entropy analysis of ocular motor noise: A case study in patients with cerebellar disease. Journal of Neuros-cience Methods, 196(2):318-326, Mar 2011.

[39] G. Veneri, P. Piu, F. Rosini, P. Federighi, A. Federico, and A. Rufa. Automatic eye fixations identification based on analysis of variance and covariance. Pattern Recogni-tion Letters, 32(13):1588 - 1593, 2011.

[40] G. Veneri, E. Pretegiani, F. Rosini, P. Federighi, A. Federico, and A. Rufa. Evaluating the human ongoing visual search performance by eye tracking application and sequencing tests. Comput Methods Programs Bio-med, Mar 2011.

[41] G. Veneri, F. Rosini, P. Federighi, A. Federico, and A. Rufa. Evaluating gaze control on a multi-target se-quencing task: The distribution of fixations is evidence of exploration optimisation. ComputBiol Med, 42(2):235-244, Feb 2012.

[42] G. Vingerhoets, F. P. de Lange, P. Vandemaele, K. Deblaere, and $\mathrm{E}$. Achten. Motor imagery in mental rotation: an fmri study. Neuroimage, 17(3):1623-1633, Nov 2002.

[43] J. M. Wolfe, M. L.-H. VГ $\mu$, K. K. Evans, and M. R. Greene. Visual search in scenes involves selective and nonselective pathways. Trends in Cognitive Sciences, In Press, Corrected Proof:0-10, 2011

[44] D. M. Wolpert. Probabilistic models in human sen-sorimotor control. Hum MovSci, 26(4):511-524, Aug 2007.

[45] R. H. Wurtz, K. McAlonan, J. Cavanaugh, and R. A. Berman. Thalamic pathways for active vision. Trends in Cognitive Sciences, In Press, Corrected Proof:0-10, 2011.

[46] J. M. Zacks, J. M. Ollinger, M. A. Sheridan, and B. Tversky. A parametric study of mental spatial trans-formations of bodies. Neuroimage, 16(4):857-872, Aug 2002.

[47] D. S. Zee, L. M. Optican, J. D. Cook, D. A. Robinson, and W. K. Engel. Slow saccades in spinocerebellardege-neration. Archives of Neurology, 33(4):243-251, Apr 1976. 